\title{
Active Opinion Maximization in Social Networks
}

\author{
Xinyue Liu \\ Department of Computer Science \\ Worcester Polytechnic Institute \\ Massachusetts, USA \\ xliu4@wpi.edu
}

\author{
Xiangnan Kong \\ Department of Computer Science \\ Worcester Polytechnic Institute \\ Massachusetts, USA \\ xkong@wpi.edu
}

\author{
Philip S. Yu \\ Department of Computer Science \\ University of Illinois at Chicago \\ Illinois, USA \\ Institute for Data Science \\ Tsinghua University \\ Beijing, China \\ psyu@uic.edu
}

\begin{abstract}
Influence maximization (IM) targets at maximizing the number of users being aware of a product by finding a set of seed users to expose in a social network. Previous IM models mainly focus on optimizing the spread of product consumption, which assumes that all users are potential customers and more exposures lead to better profit. However, in the real-world scenario, some people may not like the product and may express negative opinions after consuming, which damage the product reputation and harm the long-term profit. Only a portion of users in the social network, called the target user, is the potential customer that likes the product and will spread positive opinion. In this paper, we consider a problem called AcTive Opinion Maximization (Атом), where the goal is to find a set of seed users to maximize the overall opinion spread toward a target product in a multi-round campaign. Different from previous works, we do not assume the user opinion is known before consumption, but should be derived from user preference data. The ATOM problem has essential applications in viral marketing, such as reputation building and precision advertising. Given its significance, ATOM problem is profoundly challenging due to the hardness of estimating user opinion in a multi-round campaign. Moreover, the process of opinion estimation and influence propagation intertwine with each other, which requires the model to consider the two components collectively. We propose an active learning framework called CoNE (aCtive OpinioN Estimator) to address above challenges. Experimental results on two real-world datasets demonstrate that CONE improves the total opinion spread in a social network.
\end{abstract}

\section{CCS CONCEPTS}

- Applied computing $\rightarrow$ Economics;

\section{KEYWORDS}

Opinion Maximization; Viral Marketing; Influence Maximization; Matrix Factorization; Social Networks; Active Learning

Permission to make digital or hard copies of all or part of this work for personal or classroom use is granted without fee provided that copies are not made or distributed for profit or commercial advantage and that copies bear this notice and the full citation on the first page. Copyrights for components of this work owned by others than the author(s) must be honored. Abstracting with credit is permitted. To copy otherwise, or republish, to post on servers or to redistribute to lists, requires prior specific permission and/or a fee. Request permissions from permissions@acm.org.

KDD '18, August 19-23, 2018, London, United Kingdom

(c) 2018 Copyright held by the owner/author(s). Publication rights licensed to ACM. ACM ISBN 978-1-4503-5552-0/18/08 ..\$15.00

https://doi.org/10.1145/3219819.3220061
ACM Reference Format:

Xinyue Liu, Xiangnan Kong, and Philip S. Yu. 2018. Active Opinion Maximization in Social Networks. In Proceedings of The 24th ACM SIGKDD International Conference on Knowledge Discovery \& Data Mining (KDD '18). ACM, New York, NY, USA, 10 pages. https://doi.org/10.1145/3219819.3220061

\section{INTRODUCTION}

Motivated by applications such as viral marketing [4] and personalized recommendations [18], the problem of influence maximization $[1,3,10,13,19,20]$ has been widely studied for more than a decade. Conventional influence maximization problem aims at selecting a small set of seed users to maximize the awareness or consumption of a target product over a social network. For example, a company may select a small group of customers to distribute their trial product, hoping that they spread praises to a larger population through their social connections, which is known as the word-of-mouth effect.

Influence maximization (IM) assumes implicitly that all the users are potential customers of the product, and the more users get exposed the better market share the product will have. However, in the real-world commercial campaign, the majority of users may not like the product and may express negative opinions on it after consumption, which may damage the product reputation and harm its long-term profit. It is desirable to carefully select the seed users to avoid infecting the group of users who may spread negative opinions on the product. For example, the filmmaker of a new movie may invite a small group of audiences who are more likely to spread positive comments than negative comments in their social network to attend a preview to build a good public opinion. Opinion maximization (OM) [2, 5, 23], which aims at maximizing the total opinion generated by the activated user, is a natural variation of influence maximization. Previous studies on OM problem either randomly generate user opinions [23] or assume the user opinions are formed based on their neighbors' opinions [2, 5]. We consider to incorporate the historical opinion/rating information into opinion maximization problem. Accordingly, we study the opinion maximization problem under a new setting as follows.

Problem Studied: In an online social network, we want to maximize the total opinion expressed on a target product by selecting a set of seed users to distribute trial samples. A user may be influenced by his/her neighbors to consume the product, and the opinion towards the product is expressed based on his/her preference. We do not have any knowledge about the target product before the multi-round campaign starts, but users' historical ratings or opinions on other products are known. At the beginning of each round 


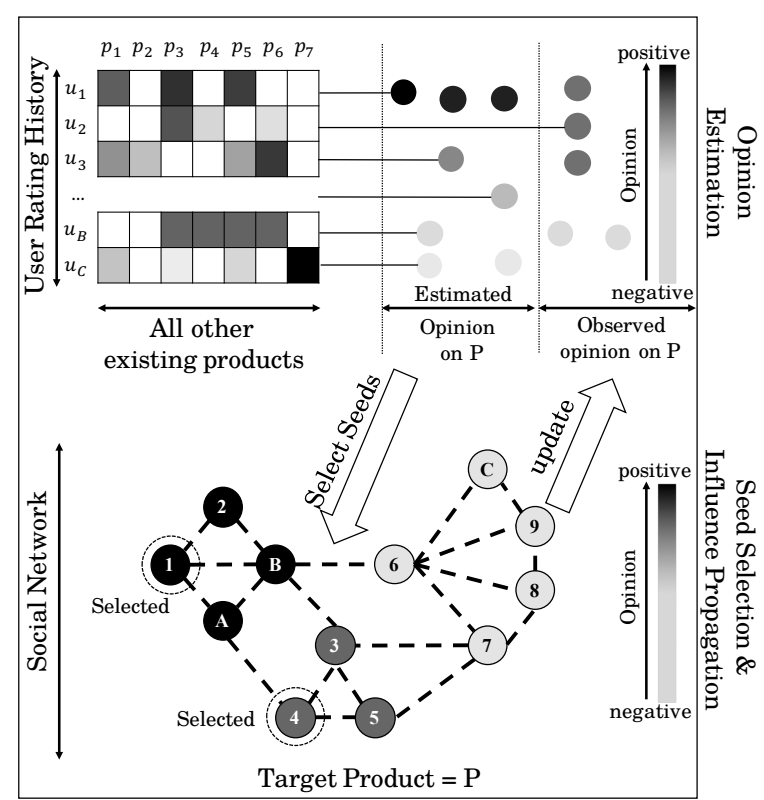

Figure 1: Example of ATOM in a social network, given a target product $P$. Different shades of grey indicate different opinions.

of the campaign, the promoter selects a set of inactive users to distribute the trial samples, and the opinion of the activated users are immediately observed. And the promoter proceeds another round until the budget is used up. We call this problem as AcTive Opinion Maximization (Атом). Fig. 1 depicts an ATOM example, where we consider a network of 12 users. The matrix on left upper side encodes the opinion observed on other products, which is used to estimate the user opinion on target product $P$. The promoter selects seeds based upon the estimation and performs the infection in the network (illustrated on the underside of Fig. 1). After the infected users express their opinions, the matrix, observed opinion on $\mathrm{P}$ and estimated opinion on $\mathrm{P}$ are updated accordingly. The promoter launches another round of seeds selection based upon the updated estimation until the budget is used up. The differences between influence maximization, opinion maximization, and Атомаre illustrated in Fig. 2. To the best of our knowledge, opinion maximization has not been studied under this context so far, and it is profoundly challenging due to the following reasons.

Negative Opinions in Majority: Conventional influence maximization methods assume implicitly that the majority of the users are potential customers. However, in the promotion of many products, such as a horror movie or Google Glass, the majority of the opinions can be detrimental due to the different preference of these users. The spread of negative opinions harms the product reputation and washes out the positive opinions. In this case, simply maximizing the spread over a social network can no longer achieve an optimal outcome. We can also illustrate this intuition of Атом from the perspective of market segmentation [17], which is an important research topic in business. For most of the products, it can be very well-received by one group of customers, while being disliked by another group of customers. The goal of market segmentation is to divide the customers in the market into several portions, and a specific marketing strategy should be investigated and designed carefully for each portion of users to maximize the profit.

Unknown Opinions: Conventional opinion maximization approaches usually assume that the opinions of the users are either known or can be generated based upon their neighbor's opinions [2, 5, 23]. However, in many real-world cases, the opinions of different users are not known for the new product being promoted. The users' opinions could either be estimated through a large amount of data on users' purchase history or their opinions can be observed once a user is exposed to the product during the influence propagation process. Because the opinion of users on the target product is unknown, conventional OM frameworks can no longer work.

Collective Opinion Estimation and Information Diffusion:

Conventional approaches on OM problem are seen as orthogonal to opinion estimation and active learning problem. However, in Атом problem, the active selection of seed nodes in information diffusion is inter-related with the estimation of user opinions. Once a seed user is selected, many other users will be exposed to the product through the social connections. The actual ratings of these users will also be expressed and observed, which affect the opinion estimation on the remaining users. Thus, the opinion estimation and seeds selection are highly related and should be considered collectively. In this paper, we link the two procedures into the multiround active seed selection setting. Under this setting, the promoter can keep selecting seeds to activate more users in multiple rounds, until using up all the seed budgets. In most of the existing works in seed user selection, only single-round propagation is considered. However, how to utilize the observed opinions to develop the optimal strategy for seeds selection under multi-round setting is still an open problem.

Hardness: The proposed Атом problem is NP-hard. With the negative user opinions, the problem is no longer sub-modular, which is different from existing IM problems [10].

To address the above challenges, we propose a novel method called aCtive OpinioN Estimator (CONE). ConE leverages the concept of active learning to select the seeds with the most opinion gain based on the estimated opinion, as illustrated in Figure 1. The contributions of this paper are summarized as follows.

- We first defined the Атом problem and proposed the first solution for it. The proposed Cone framework exploits the historical rating data to facilitate the opinion estimation on the target product. To the best of our knowledge, this work is the first effort to utilize the real-world historical ratings to improve the performance of opinion maximization.

- We filled the gap between opinion maximization and item recommendation (i.e., rating prediction) by leveraging the concept of active learning.

- We conducted extensive experiments on two real-world social networks associated with historical rating data. The experimental results show that our method can largely improve the total opinion spread in the network. 


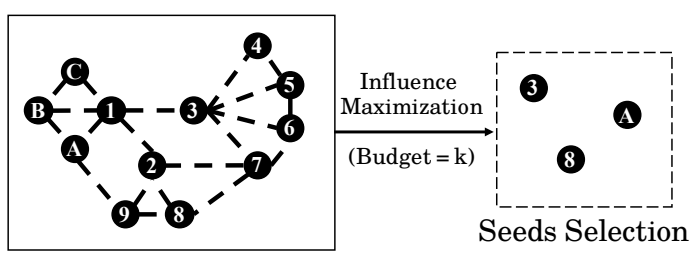

Social Network

(a) Influence Maximization [10]

Target product with known properties

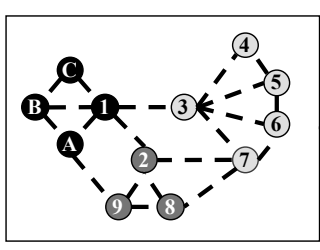

Social Network

(b) Opinion Maximization [2]

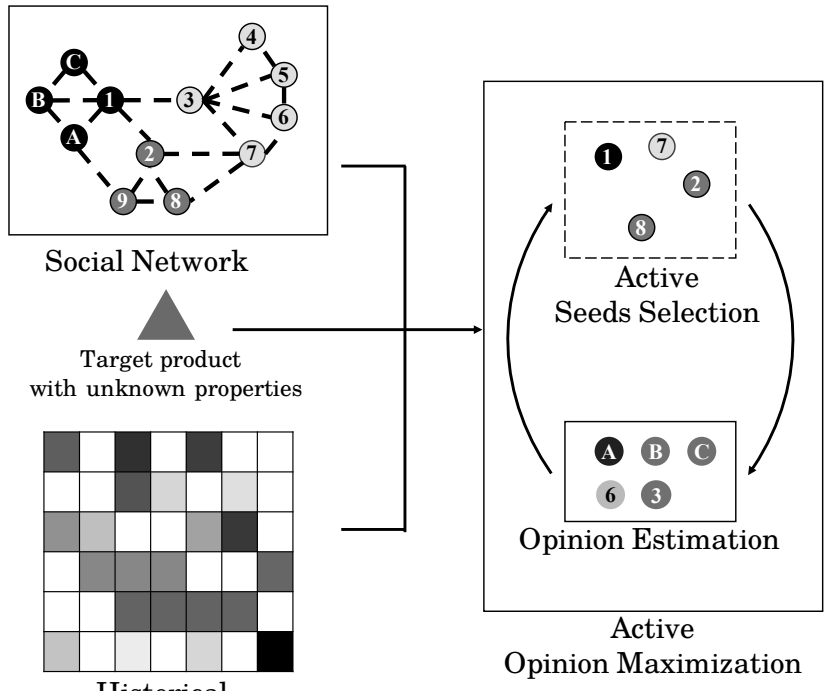

Historical

Rating Matrix

(c) Active Opinion Maximization (Proposed CoNE) [This paper]

Figure 2: Comparison of Influence Maximization (IM), Opinion Maximization (OM) and Active Opinion Maximization (AтoM). Different shades of grey indicate different opinions. IM does not distinguish opinions and aims at maximizing the spread of product consumption; $O M$ assumes that the opinion on target product is known or can be inferred before the campaign; Aтомutilizes the observed opinion data and actively estimate user opinion towards target product in a multi-round campaign.

\section{PROBLEM FORMULATION}

\subsection{Notations}

We use lower case letters (e.g., $x$ ) to denote scalars, lower case bold letters (e.g., $\boldsymbol{x})$ to denote column vectors, bold-face upper case letters (e.g., X) to denote matrices, upper case letters (e.g., X) to denote elements of matrices and upper case calligraphic letters $(e . g ., \mathcal{X})$ to denote sets. We use $\mathrm{X}(i,:)$ (and $\mathrm{X}(:, j))$ to denote the $i$-th row (and the $j$-th column) of X. The projection of a matrix $X$ onto domain $\mathcal{S}$ is denoted by $P_{\mathcal{S}}(\mathrm{X})$.

\subsection{Concepts Definition}

DEFinition 2.1 (SOCIAL NETWORK): An online social network can be represented as a directed graph $G=(\mathcal{V}, \mathcal{E}, \mathcal{W})$, where $\mathcal{V}=$ $\left\{u_{1}, \ldots, u_{m}\right\}$ is the set of users (nodes), $\mathcal{E}=\left\{e_{i j} \mid i=1, \ldots, m ; j=\right.$ $1, \ldots, m\} \subseteq \mathcal{V} \times \mathcal{V}$ is the set of social links (edges) among users in $\mathcal{V}, \mathcal{W}=\left\{w_{i j} \mid i=1, \ldots, m ; j=1, \ldots, m\right\}$ is the set of link weights and $w_{i j}$ denotes the weight of link $e_{i j}$. The set of items (products) associated with $G$ is denoted as $\mathcal{P}=\left\{p_{1}, \ldots, p_{n}\right\}$. Let $\mathcal{R}=\left\{r_{i j} \in \mathbb{R} \mid i=1, \ldots, m ; j=1, \ldots, n\right\}$ be the set of observed ratings, where $r_{i j}$ denotes the rating on product $p_{j}$ assign by user $u_{i}$. Let $\Omega=\left\{(i, j) \mid r_{i j} \in \mathcal{R}\right\}$ be the set of observed rating indices. Higher rating value indicates better opinion.

Definition 2.2 (RATING MAtrIX): The set of observed ratings $\mathcal{R}$ can be cast into a sparse rating matrix $\mathbf{R} \in \mathbb{R}^{(m \times n)}$ where $R_{i j}=r_{i j}$ for all $(i, j) \in \Omega$ and $R_{i j}=0$ for all $(i, j) \notin \Omega$. In other words, $R_{i j}=0$ indicates that the rating on product $p_{j}$ assign by user $u_{i}$ is unobserved.
Definition 2.3 (OPINION): $o_{i j}=r_{i j}-r_{\text {neutral }} \in \mathbb{R}$ is the opinion given by user $u_{i}$ on the product $p_{j}$, where $r_{\text {neutral }}$ is the rating value corresponds to the neutral opinion.

Definition 2.4 (USER Profile): User profile for $u_{i}$ is a $\kappa$-dimensional vector $\boldsymbol{u}_{i} \in \mathbb{R}^{\kappa}$ which indicates the characters of $u_{i}$.

Definition 2.5 (ITEM PROFILE): Item profile for $p_{j}$ is a $\kappa$-dimensional vector $\boldsymbol{v}_{j} \in \mathbb{R}^{\kappa}$ which indicates the characters of $p_{j}$.

Definition 2.6 (Multi-Round LiNEAR THRESHOLD): Multi-round Linear Threshold (MLT) is a multi-round diffusion model. Similar to the conventional LT model [10], in which each user $u_{i}$ in a weighted social network $G$ is associated with a threshold $\theta_{i} \in[0,1]$. Before the first round of MLT, all users are inactive towards the target product. At round $t$ of the MLt, an inactive user $u_{i}$ becomes active when $\sum_{u_{j} \in C^{(t-1)}} w_{j i}>\theta_{i}$, where $C^{(t-1)}$ is the set of active users before round $t$ starts. At each round, the influence only propagates $\ell$ layers of neighbors before the next round starts, where $\ell \geq 1$ is a model parameter. All active users will stay active and cannot back to inactive status.

DEFINITION 2.7 (MARKETING STRATEGY): In this paper, we are mainly concerned about the seed user selection problem. For simplicity, we refer to the marketing strategy of the target product $p_{t}$ as the vector of seed users sets $S=\left(\mathcal{S}^{(1)}, \ldots, \mathcal{S}^{(T)}\right)$, where $\mathcal{S}^{(q)}$ is the set of seed users been selected for round $q$, and $\mathrm{T}$ the terminal round number. 


\subsection{Problem Definition}

Definition 2.8 (ACTIVE OPINION MAXIMIZATION): Given a social network $G$, an item set $\mathcal{P}$, a rating matrix $\mathbf{R}$, a target product $p_{t} \notin \mathcal{P}$, the terminal round number $T$ and the MLT diffusion model, the goal of Атом is to select $k^{(q)}$ seeds from $\mathcal{V}$ to activate at the beginning of round $q$ to maximize the total opinion given by active users in $G$ towards $p_{t}$ at the end of round $T$. Accordingly, the proposed problem can be formulated as

$$
\begin{aligned}
& \underset{\mathcal{S}^{(1)}, \ldots, \mathcal{S}^{(T)}}{\operatorname{maximize}} I_{\ell}\left(\mathcal{S}^{(0)}, \ldots, \mathcal{S}^{(T)}\right) \\
& \text { s.t. }\left|\mathcal{S}^{(q)}\right|=k^{(q)}, \mathcal{S}^{(q)} \subseteq \mathcal{V} \backslash C^{(q-1)}
\end{aligned}
$$

where $I_{\ell}: S \rightarrow \mathbb{R}$ is the influence function that maps the sequence of seed user sets to the total opinion of the final active users. Since the opinion given by the active users is unknown before the propagation starts, the mapping function $I_{\ell}$ cannot be explicitly defined. Note that once $u_{i}$ is activated, his/her opinion $o_{i} \in \mathbb{R}$ on $p_{t}$ is observed, and $o_{i}$ cannot change once been expressed. After the propagation finishes, the final set of active users can be denoted as $C^{(T)}$. Then the total opinion can be computed as $\sum_{u_{i} \in C^{(T)}} o_{i}$.

In this paper, we do not assume any prior knowledge about the target product $p_{t}$ is given before the propagation starts, which simulates the most general case of viral marketing where a brandnew product is being promoted in a social network.

Given the conventional influence maximization on LT model is $\mathrm{NP}$-hard [10], the proposed multi-round opinion maximization is also NP-hard. Kempe et al. proved that with LT model, although IM problem is NP-hard, it is monotone and submodular, so the greedy algorithm proposed in [10] can achieve $\left(1-\frac{1}{e}\right)$-approximation of the optimal seeds selection. However, in our proposed problem, the negative opinion generated by the activated users can cancel out the positive opinion and harm the final outcome. So the proposed problem is no longer submodular or monotone, and the conventional greedy algorithm cannot guarantee any lower bound on the approximation even if all the opinions of users are given before the propagation. Furthermore, the opinion of users on target product is unknown in Атом, which makes it even more challenging to solve.

\section{METHODOLOGY}

In this section, we introduce the proposed method Cone in details. We first briefly review the low-rank matrix factorization method. Then we propose an approach to estimate the profile vector for the target item. Based on the estimated ratings, we present a greedy algorithm for maximizing the total opinion under MLT diffusion model. Finally, we put everything together to propose the CONE algorithm.

\subsection{Low-Rank Matrix Factorization}

We first review the matrix factorization method for collaborative filtering [12]. The goal of collaborative filtering is to predict unknown ratings based on observed ratings in $\mathbf{R}$. Specifically, we associate a $\kappa$-dimensional vector $\boldsymbol{u}_{i} \in \mathbb{R}^{\kappa}$ for each user $u_{i}$ and $\boldsymbol{v}_{j} \in \mathbb{R}^{\kappa}$ for each item $p_{j}$. The vector $\boldsymbol{u}_{i}$ and $\boldsymbol{v}_{j}$ are usually called user profile and item profile since they capture the characteristics of users and items, as defined in Sec. 2.2. The rating $R_{i j}$ of user $u_{i}$ towards item

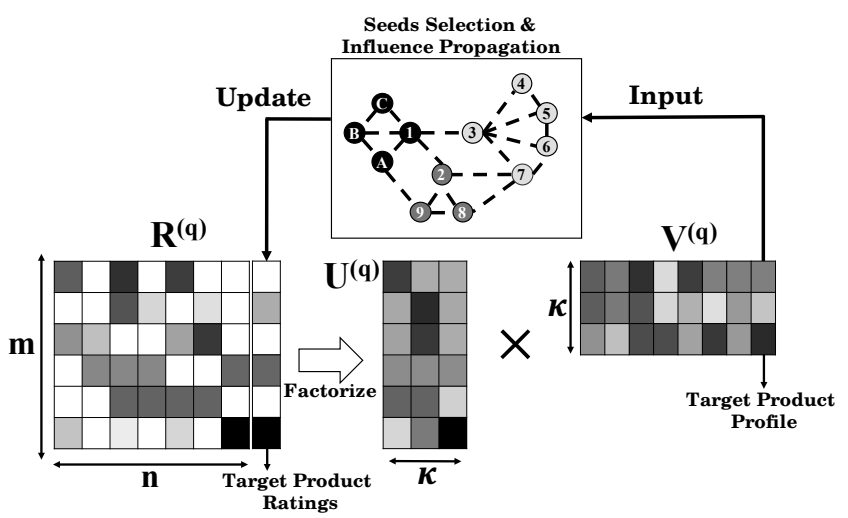

Figure 3: Matrix factorization for actively learning the target product profile. Different shades of grey indicate different opinion.

$p_{j}$ can be approximated by the inner product of $\boldsymbol{u}_{i}$ and $\boldsymbol{v}_{j}$, which is $\boldsymbol{u}_{i}^{\top} \boldsymbol{v}_{j}$

Given the set of observed ratings $\Omega$, the user profiles and item profiles can be learned through fitting the observed ratings by solving the following optimization problem:

$$
\underset{\mathbf{U}, \mathbf{V}}{\operatorname{minimize}}\left\|P_{\Omega}\left(\mathbf{R}-\mathbf{U}^{\top} \mathbf{V}\right)\right\|_{F}^{2}+\lambda\left(\|\mathbf{U}\|_{F}^{2}+\|\mathbf{V}\|_{F}^{2}\right),
$$

where $\mathbf{U}=\left(\boldsymbol{u}_{1}, \ldots, \boldsymbol{u}_{m}\right) \in \mathbb{R}^{(\kappa \times m)}, \mathbf{V}=\left(\boldsymbol{v}_{1}, \ldots, \boldsymbol{v}_{n}\right) \in \mathbb{R}^{(\kappa \times n)}$, and the projection $P_{\Omega}(\mathrm{X})$ is the matrix with only the indices in $\Omega$ of $\mathrm{X}$ preserved. Besides, $\|\cdot\|_{F}^{2}$ denotes the Frobenius 2-norm and $\lambda \geq 0$ is the regularization parameter for avoiding over-fitting. The problem can be solved by exiting numerical optimization methods such as alternating minimization or stochastic gradient descent. Alternating minimization method performs the following steps to obtain the final estimation of $\mathbf{U}$ and $\mathbf{V}$.

Step 1: Randomly initialize $\mathbf{U} \in \mathbb{R}^{(\kappa \times m)}$ and $\mathbf{V} \in \mathbb{R}^{(\kappa \times n)}$.

Step 2: For $j=1, \ldots, n$, minimizing with respect to $\boldsymbol{v}_{j}$ with $\mathrm{U}$ fixed:

$$
\underset{\boldsymbol{v}_{j}}{\operatorname{minimize}}\left\|\mathbf{R}\left(\Omega^{j},:\right)-\mathbf{U}\left(:, \Omega^{j}\right)^{\top} \boldsymbol{v}_{j}\right\|_{F}^{2}+\lambda\left\|\boldsymbol{v}_{j}\right\|_{2}
$$

where $\Omega^{j}=\{i \mid(i, j) \in \Omega\}$. Eq. 3 is essentially a ridge regression problem, which has a closed form solution as follows:

$$
\hat{\boldsymbol{v}}_{j}=\left(\mathbf{U}\left(:, \Omega^{j}\right) \mathbf{U}\left(:, \Omega^{j}\right)^{\top}+\lambda \mathbf{I}\right)^{-1} \mathbf{U}\left(:, \Omega^{j}\right) \mathbf{R}\left(\Omega^{j},:\right)
$$

By solving $n$ separate regression problems for each $\boldsymbol{v}_{j}$, we can obtain an estimation of $\mathbf{V}$.

Step 3: Symmetrically, with V fixed we can find U by solving $m$ separate ridge regression problems:

$$
\underset{\boldsymbol{u}_{i}}{\operatorname{minimize}}\left\|\mathbf{R}\left(:, \Omega_{i}\right)-\boldsymbol{u}_{i}^{\top} \mathbf{V}\left(:, \Omega_{i}\right)\right\|_{F}^{2}+\lambda\left\|\boldsymbol{u}_{i}\right\|_{2}
$$

where $\Omega_{i}=\{j \mid(i, j) \in \Omega\}$. The closed form solution for $\boldsymbol{u}_{i}$ is given by:

$$
\hat{\boldsymbol{u}}_{i}=\left(\mathbf{V}\left(:, \Omega_{i}\right) \mathbf{V}\left(:, \Omega_{i}\right)^{\top}+\lambda \mathbf{I}\right)^{-1} \mathbf{V}\left(:, \Omega_{i}\right) \mathbf{R}\left(:, \Omega_{i}\right)
$$

Step 4: Perform step (2) and step (3) alternatively until the estimation of $\mathrm{U}$ and $\mathrm{V}$ converges.

Output: Finally, the estimated rating matrix $\hat{R}=U^{\top} \mathbf{V}$, where the missing ratings in $\mathbf{R}$ are filled. The estimated $\mathbf{U}$ and $\mathbf{V}$ are called user profile matrix and item profile matrix respectively. 


\subsection{Learning Profile of Target Item}

Since higher rating indicates a better opinion, we can extract information about user opinion based on the historical ratings. In multi-round opinion maximization problem, we aim at finding the best seed users at the beginning of each round to maximize the total opinion on the target item. It is usually the case that the target item is new to the market and nobody has reviewed or rated it yet. Thus, it is challenging to estimate the user opinion on the target item. Given a newly emerging target item (product) $p_{t}$ in a social network $G$, a simple approach to build the profile for it is to first randomly generate a $\kappa$-dimensional vector $\boldsymbol{v}_{t}^{(0)} \in \mathbb{R}^{\kappa}$. At the end of round $q$ of the proposed problem, a set of new ratings can be observed on $p_{t}$, and the item profile can be updated accordingly to obtain $\boldsymbol{v}_{t}^{(q)}$.

Suppose that $\boldsymbol{r}^{(q)} \in \mathbb{R}^{m}$ denotes the observed rating vector for $p_{t}$ before round $q$ starts, where $r_{i}^{(q)}$ refers to the observed rating given by $u_{i}$ on $p_{t}$. Note that $r_{i}^{(q)}=0$ indicates that the rating of $p_{t}$ given by $u_{i}$ is not yet observed.

At round $q$, we can attach the observed rating vector $\boldsymbol{r}^{(q)}$ to the historical rating matrix $\mathbf{R}$ to construct a new matrix $\mathbf{R}^{(q)}=$ $\left(\mathbf{R}, \boldsymbol{r}^{(q)}\right) \in \mathbb{R}^{(m \times(n+1))}$. The updated user profiles and item profiles can be derived by solving the following optimization:

$\underset{\mathbf{U}^{(q)}, \mathbf{V}^{(q)}}{\operatorname{minimize}}\left\|P_{\Omega}\left(\mathbf{R}^{(q)}-\left(\mathbf{U}^{(q)}\right)^{\top} \mathbf{V}^{(q)}\right)\right\|_{F}^{2}+\lambda\left(\left\|\mathbf{U}^{(q)}\right\|_{F}^{2}+\left\|\mathbf{V}^{(q)}\right\|_{F}^{2}\right)$

Thus, $\mathbf{U}^{(q)} \in \mathbb{R}^{(m \times \kappa)}$ is the updated user profile matrix and $\mathbf{V}^{(q)} \in \mathbb{R}^{(\kappa \times(n+1))}$ is the the updated item profile matrix at the end of round $q$. The last row of $\mathbf{V}^{(q)}$ is the updated profile vector for the target item $p_{t}$, that is $\boldsymbol{v}_{t}^{(q)}=\mathrm{V}^{(q)}(:, n+1)$. Now we can use $\mathrm{U}^{(q)}$ and $v_{t}^{(q)}$ to refine the predictions on users' opinion of $p_{t}$, which can be done by simply computing the following inner product:

$$
\hat{\boldsymbol{r}}^{(q)}=\left(\mathrm{U}^{(q-1)}\right)^{\top} \boldsymbol{v}_{t}^{(q-1)},
$$

where $\hat{\boldsymbol{r}}^{(q)} \in \mathbb{R}^{m}$ denotes the prediction of user opinion on $p_{t}$ at the end of round $q$. Obviously, as we observe more ratings given by the activated users in the network, the approximation of $U^{(q)}$ and $\boldsymbol{v}_{t}^{(q)}$ will be more accurate, which leads to a better prediction towards $\hat{\boldsymbol{r}}^{(q)}$.

The item profile learning approach is also illustrated in Figure 3. In the rating matrix $\mathbf{R}$ on the left side in Figure 3, each column refers to the ratings of a product. We use the rightmost column of $\mathrm{R}$ to denote the ratings of the target item $p_{t}$. Note that $\mathrm{R}$ is partially observed and usually very sparse. In iteration $q$ of the algorithm, we first factorize the rating matrix $\mathbf{R}^{(q)}$ to get two profile matrices $\mathrm{U}^{(q)}$ and $\mathrm{V}^{(q)}$ (illustrated as the two matrices on the right side in Figure 3), where $U^{(q)}$ stores the current profile for users and $\mathbf{V}^{(q)}$ stores the current profile of items. And it is easy to see the last column of $\mathbf{V}^{(q)}$ is the profile vector of $p_{t}$ in iteration $q$, which is also denoted as $\boldsymbol{v}_{t}^{(q)}$. Using $\mathrm{U}^{(q)}$ and $\boldsymbol{v}_{t}^{(q)}$, we can estimate the user's opinions towards $p_{t}$ using Eq. 8. With the estimation of opinions, our algorithm can greedily select the best $k^{(q)}$ inactive users to activate, and the detailed selection strategy will be discussed in Sec. 3.3. As the influence of the newly activated users propagates, more users will be activated and express their opinions towards $p_{t}$. And these newly observed opinions are added to the last column of $\mathbf{R}^{(q)}$ to get the rating matrix $\mathbf{R}^{(q+1)}$ for next iteration. Then we perform the same steps as above to obtain $\mathrm{U}^{(q+1)}$ and $\mathrm{V}^{(q+1)}$. As we observe more opinions generated by users, our algorithm can gradually approach more and more accurate estimations of the user profile and item profile.

\subsection{Greedy Algorithm on Opinion Maximization}

We have proposed an active approach to estimate the opinion of users in the network while the influence is propagated, the remaining question is how to select the best set of seed users based on the opinion estimation at each round of MLT. Although the original problem aims at selecting seed users for the round $1, \ldots, T$ of the MLt diffusion model, it is obvious that the seed selection of the round $q$ is depended on the final propagation results of the round $q-1$. Thus, the proposed Атом problem can be broken down into $T$ separate sub-problem of opinion maximization where each sub-problem is depended on the output of previous one. Inspired by [10], we propose an analogous greedy algorithm to solve each sub-problem. The pseudo-code of the algorithm is available in Alg. 1.

Suppose we are at the round $q$ of the Атом problem. Before current round starts, we have obtained the activated users set $C^{(q-1)}$ as well as their ratings on the target items, which can be denoted by $\boldsymbol{r}^{(q-1)}$. The problem of opinion maximization in the round $q$ can be formulated as:

$$
\begin{aligned}
& \underset{\mathcal{S}^{(q)}}{\operatorname{maximize}} \bar{I}_{\ell}\left(\mathcal{S}^{(q)} \cup C^{(q-1)}\right) \\
& \text { s.t. }\left|\mathcal{S}^{(q)}\right|=k^{(q)}, \mathcal{S}^{(q)} \subseteq \mathcal{V} \backslash C^{(q-1)}
\end{aligned}
$$

where $\bar{I}_{\ell}: \mathcal{S} \rightarrow \mathbb{R}$ is the opinion function that maps a set of seed users to the total opinion achieved at the end of the current round. The predicted ratings $\hat{\boldsymbol{r}}^{(q)}$ can be inferred using the approach proposed in Sec. 3.2. Given the seed budget $k^{(q)}$, we can greedily select $k^{(q)}$ users who can maximize the total opinions of the final activated users. To do so, we scan all the inactive users in the network $k^{(q)}$ times. In every scan, we add each inactive user to the temporary seed set $\mathcal{S}$ and propagate the influence to obtain the final activated users. Then we can compute the estimated total opinion of these activated users using the mapping function $\bar{I}_{\ell}$ and $\hat{\boldsymbol{r}}^{(q)}$. Among all $u$ in the scan, the one with the largest estimated opinion is added to $\mathcal{S}$. We repeat the scan until run out of budget or there are no more inactive users in the network.

\subsection{The CONE Method}

Now we can put everything together to present the CONE algorithm. The pseudo-code of CONE algorithm is summarized in Alg. 2. The CONE algorithm includes the following steps:

(1) Initialization: Given the partially observed rating matrix $\mathbf{R}$, we firstly estimated the user profile matrix $\mathbf{U}^{(0)}$ by solving the Eq. 7 with $q=0$, where the parameter $\lambda$ and $\kappa$ can be selected via crossvalidation to minimize the prediction error. Although we can obtain $\mathbf{V}^{(0)}$ by solving Eq. 7, it does not contain profile for the target item 


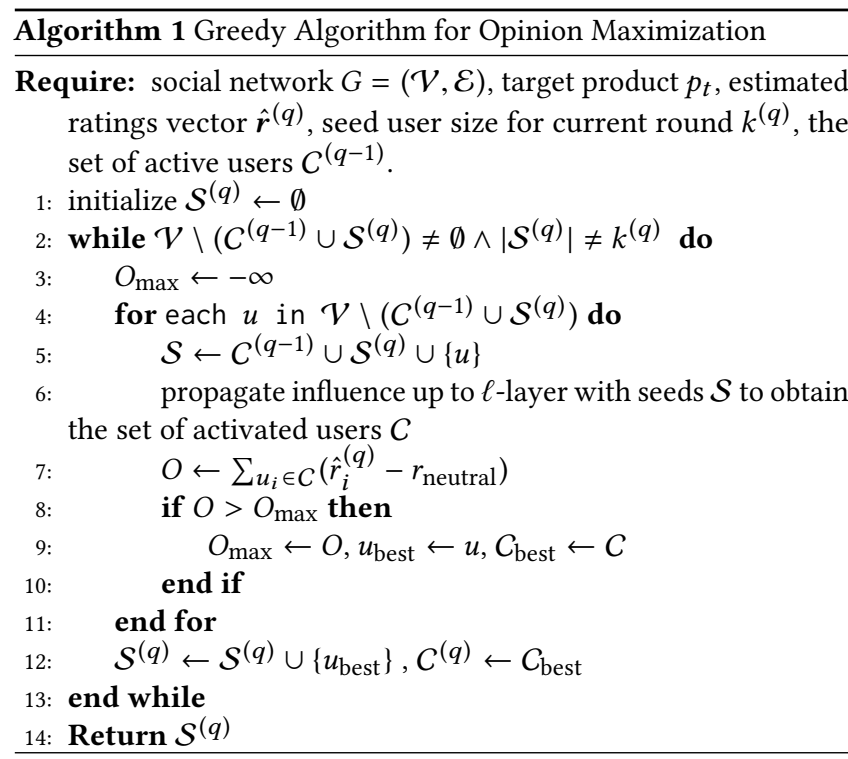

$p_{t}$ since $\mathbf{R}$ does not have ratings of $p_{t}$. Then we randomly initialize the profile vector of target product $\boldsymbol{v}_{t}^{(0)}$.

(2) Rating Estimation:At the beginning of round $q$, we first update the estimated ratings $\hat{\boldsymbol{r}}^{(q)}$ for $p_{t}$ using Eq. 8.

(3) Seeds Selection: Then we use $\hat{\boldsymbol{r}}^{(q)}$ and $C^{(q-1)}$ as the input for Algorithm 1 to select $k^{(q)}$ best users to maximize the estimated total opinions at the end of round $q$. The set of seed users selected in this round is $\mathcal{S}^{(q)}$.

(4) Profile Update: As $\mathcal{S}^{(q)}$ are activated, the real influence is propagated and the newly activated users express their opinions on the target product. Let $C^{(q)}$ be the updated set of activated users. We user the opinions of $C^{(q)}$ to update the observed rating vector $\boldsymbol{r}^{(q)}$. Let $\mathbf{R}^{(q)}=\left(\mathbf{R}, \boldsymbol{r}^{(q)}\right)$ be the new extended rating matrix, which is used as the rating matrix to infer the user profile matrix $\mathrm{U}^{(q)}$ and item profile matrix $\mathbf{V}^{(q)}$ for next round by solving Eq. 7. As we showed in Sec. 3.2, the last column of updated profile matrix $\mathbf{V}^{(\mathbf{q})}$ is the item profile of $p_{t}$, which is denoted as $\boldsymbol{v}_{t}^{(q)}$.

(5) Repeat: Cone repeats step (2)-(4) until it reaches the terminal round number $T$ or there are no more inactive users in the network. (6) Output: The output of the Cone is the vector of seed users selected for each round, i.e., $\left(\mathcal{S}^{(1)}, \ldots, \mathcal{S}^{(T)}\right)$.

\subsection{Complexity Analysis}

The time complexity of each iteration of Cone is about $O\left(m^{2}+(m+\right.$ $\left.n) \kappa^{3}\right)$, the first term and the second term is the cost of line 8 and line 11 of Algorithm 2 respectively, and they dominate other operations. In the factorization model, the number of factors $\kappa$ is usually much smaller than the number of users $m$ and the number of items $n$ (i.e., $\kappa \ll \min (m, n))$, and the best choice of $\kappa$ does not change too much while $m$ or $n$ increases. And the number of rounds/iterations (T) of CONE is also relatively small. For all the datasets we tested in the experiments, the network can be fully activated within 10 to 50 iterations. Hence, the time complexity is majorly dominated by the greedy search operation, which can be simply denoted as $O\left(\mathrm{~m}^{2}\right)$.

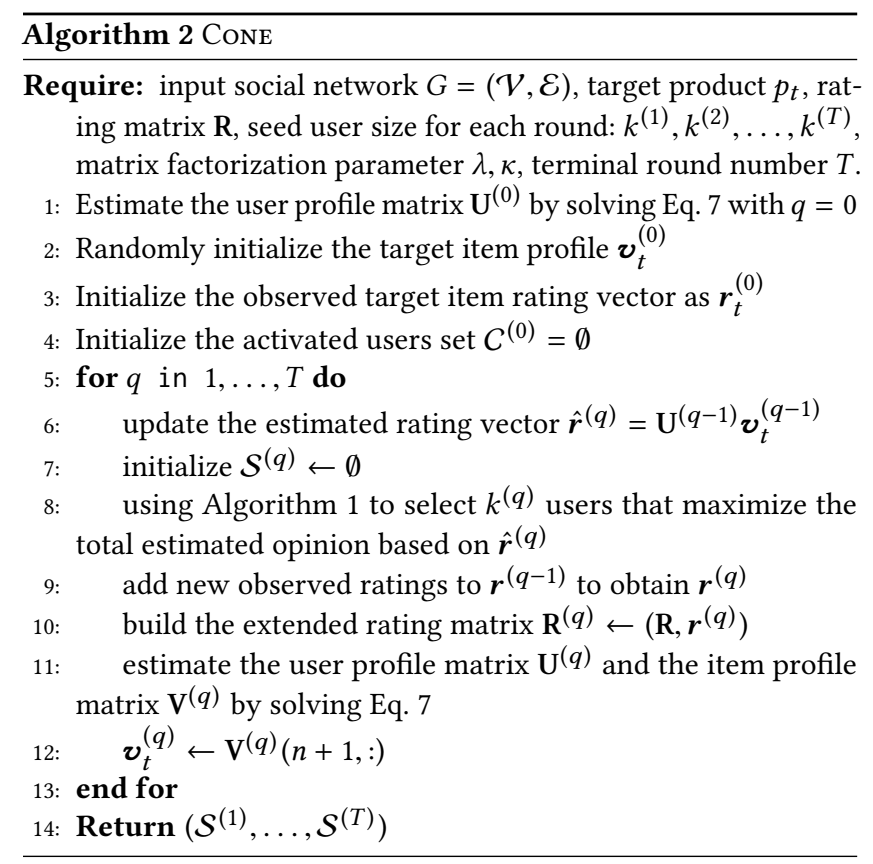

For the space complexity, ConE needs to store two profile matrices with complexities of $O(m \kappa)$ and $O(n \kappa)$, a preference matrix with complexity of $O(m n)$ and the social network with complexity of $O\left(m^{2}\right)$. Hence, the total space complexity is $O\left(m^{2}+m n+(m+n) \kappa\right)$, which is approximately $O\left(m^{2}+m n\right)$. Considering that the adjacency matrix of the network and the preference matrix are usually very sparse, the actual space complexity is much lower than it.

\section{EXPERIMENTS}

To test the effectiveness of the proposed model, we have conducted extensive experiments on real-world networks. In this section, we will first introduce the datasets and the detailed experiment settings. After that, we will show the experimental results together with the analysis and discussion.

\subsection{Datasets}

To evaluate the performance of the proposed approach for opinion maximization, we tested our method on two real-world datasets with networks and historical ratings.

1) Flixster [9]: Flixster ${ }^{1}$ is one of the main players in the mobile and social movie rating business. This dataset was crawled by the authors of [9], which contains 137,925 nodes and 1,269,373 edges. A collection of historical ratings are associated with the network, which contains the ratings of 48,794 items given by the users in the network. Since it is difficult to handle the original size of the dataset, we sample a smaller network for our experiments. We follow [6] to use Graclus $^{2}$ to obtain a subgraph by means of graph clustering. We filter out items with less than five ratings from all the users in the sampled subgraph.

\footnotetext{
${ }^{1}$ http://www.flixster.com

${ }^{2}$ http://www.cs.utexas.edu/users/dml/Software/graclus.html
} 
Table 1: Statistics of Datasets.

\begin{tabular}{c|ccccc}
\hline & \# Nodes & \# Edges & \# Items & \# Ratings & Linkage \\
\hline Flixster & 5,372 & $58 \mathrm{k}$ & 3,470 & $110 \mathrm{k}$ & undirected \\
CiaoDVD & 4,658 & $40 \mathrm{k}$ & $16 \mathrm{k}$ & $72.6 \mathrm{k}$ & directed \\
\hline
\end{tabular}

2) CiaoDVD [7]: CiaoDVD is a dataset crawled from the entire category of DVDs from the price comparison and product review website Ciao! ${ }^{3}$. Trust links exist in CiaoDVD dataset reflects the trust relationship between users. We view such links as the (directed) edges of the network. As we described above, we removed items with less than five ratings. The statistics of datasets used in our experiments are also summarized in Table 1.

\subsection{Compared Methods}

The detailed information about the comparison methods are listed as follows:

- Cone: Cone is the proposed method based on the Mrt diffusion model.

- CONE w/o net: CONE w/o net (or CONE-) is a degenerated version of CONE, which selects the users with highest predicted opinion on the target items as the seeds at each round. CONE- does not consider the propagation model and works like a recommendation model that tries to activate the users with the highest expected opinion.

- LTRANdom [10]: Method LTRAndom is a baseline method which randomly selects inactive nodes as the seeds.

- LTDEgreE [21]: Method LTDEgrEe is another baseline method which selects the nodes with highest degree at the beginning of each round in MLt. The degree heuristics are commonly used in the sociology literature as the estimates of a node's influence [21].

- LTgreedy [10]: Method LTgreedy is an influence maximization method for linear threshold model proposed in [10]. We adapt the original algorithm to fit the multi-round setting.

\subsection{Experiment Setup}

The social links among users in Flixster dataset are undirected, but in CiaoDVD they are directed. To unify different kinds of networks in our model, we replace undirected links, e.g., $u_{i}-u_{j}$, with two directed links $u_{i} \rightarrow u_{j}$ and $u_{i} \leftarrow u_{j}$. In MLt diffusion model, a user $u_{i}$ can influence his neighbors with specific influence weights and has a threshold $\theta_{i}$, which denotes the minimal required influence to be activated by the neighbors. The weights of the directed social links $e_{i j}$ quantify the influence propagates from $u_{i}$ to $u_{j}$. Following $[19,24]$, we quantify the weight of $e_{i j}$ using Jaccard Coefficient: $w_{i j}=\frac{\left|\Gamma_{\text {in }}\left(u_{i}\right) \cap \Gamma_{\text {out }}\left(u_{j}\right)\right|}{\left|\Gamma_{\text {in }}\left(u_{i}\right) \cup \Gamma_{\text {out }}\left(u_{j}\right)\right|}$, where $\Gamma_{\text {in }}\left(u_{i}\right)$ is the set of users who follow $u_{i}\left(e . g ., u \rightarrow u_{i}\right)$ and $\Gamma_{\text {out }}\left(u_{i}\right)$ is the set of users who are followed by $u_{i}$ (e.g., $u_{i} \rightarrow u$ ). Following $[23,24]$, we randomly generate the set of threshold of users $\left\{\theta_{1}, \ldots, \theta_{m}\right\}$ from uniform distribution within range $[0,1]$. Due to the rating matrix is incomplete in all datasets, we first use low-rank matrix factorization described in Sec. 3 to fill the missing values in $\mathrm{R}$. To get the best approximation of the ground truth, the values of $\kappa$ and $\alpha$ used in the final factorization

\footnotetext{
${ }^{3}$ http://ciao.co.uk
}

Table 2: \# pos. opinion v.s. \# neg. opinion (avg. \# (rank)), $\uparrow$ indicates higher is better, $\downarrow$ indicates lower is better.

\begin{tabular}{c|cccc}
\hline Methods & \# pos. $(\uparrow)$ & \# neg. $(\downarrow)$ & \# pos. - \# neg. ( $\uparrow)$ & rank \\
\hline & \multicolumn{4}{|c}{ Flixster } \\
\hline LTRANDOM & $1278.00(4)$ & $1146.92(4)$ & $170.67(4)$ & 4.00 \\
LTDEGREE & $1274.00(5)$ & $1109.00(3)$ & $165.00(5)$ & 4.33 \\
LTGREEDY & $1363.08(1)$ & $1277.30(5)$ & $216.15(2)$ & 2.67 \\
CoNE- & $1283.16(3)$ & $1082.50(1)$ & $200.63(3)$ & 2.33 \\
CONE & $1328.00(2)$ & $1098.88(2)$ & $229.13(1)$ & $\mathbf{1 . 6 7}$ \\
\hline & \multicolumn{4}{|c}{ CiaoDVD } \\
\hline LTRANDOM & $648.09(5)$ & $451.40(2)$ & $196.61(5)$ & 4.00 \\
LTDEGREE & $749.66(2)$ & $544.46(4)$ & $205.22(3)$ & 3.00 \\
LTGREEDY & $788.85(1)$ & $588.27(5)$ & $200.65(4)$ & 3.33 \\
CONE- & $658.44(4)$ & $430.84(1)$ & $227.61(2)$ & 2.33 \\
CONE & $743.01(3)$ & $490.61(3)$ & $252.47(1)$ & 2.33 \\
\hline
\end{tabular}

are selected via 5 -fold cross-validation. We denote the filled rating matrix as $\overline{\mathbf{R}}$. Thus, $\bar{R}_{i j}$ denotes the ground truth rating on item $p_{j}$ given by $u_{i}$. To distinguish positive/negative opinions, we convert the ratings in $\overline{\mathbf{R}}$ to opinion as:

$$
o_{i j}= \begin{cases}r_{h}-r_{\mathrm{avg}}, & \text { if } \bar{R}_{i j}>r_{h} \\ r_{l}-r_{\mathrm{avg}}, & \text { if } \bar{R}_{i j}<r_{l} \\ \bar{R}_{i j}-r_{\mathrm{avg}}, & \text { otherwise }\end{cases}
$$

where $r_{h}$ and $r_{l}$ are the highest rating and lowest rating allowed in the corresponding dataset respectively, and $r_{\text {avg }}$ is the average value of all ratings in $\overline{\mathbf{R}}$. In other words, we consider $r=r_{\mathrm{avg}}$ as the neutral opinion, $r>r_{\mathrm{avg}}$ as positive opinion and $r<r_{\mathrm{avg}}$ as negative opinion.

For each experiment, we randomly select an item from $\mathcal{P}$ as the target item and remove the corresponding column in $\mathbf{R}$ to obtain $\tilde{\mathbf{R}} \in \mathbb{R}^{(m \times(n-1))}$. Our model uses $\tilde{\mathbf{R}}$ as the observed historical rating matrix to infer the preference of users on the target item during the diffusion. We follow [13] to set $\ell=1$ for all the experiments. And the total budget of seed users are all set as 50 , we let $k^{(1)}=k^{(2)}=$ $\cdots=k^{(q)}=k$ where $k$ is the diffusion model parameter denotes the number of seeds to be selected in each round. We vary $k$ to get different diffusion results. We obtain the total opinion of the final set of activated users using $\overline{\mathbf{R}}$ and Eq. 10. We repeat the target product selection 50 times and report the average total opinion. The parameter $\lambda$ and $\kappa$ used in CONE and CONE- are tuned using 10 -fold cross validation.

\subsection{Performance on Opinion Maximization}

The results of compared methods in terms of total opinion achieved are summarized in Figure 4. In Figures 4(a)-(c) we demonstrate the total opinion achieved by all compared methods on Flixster dataset with $k=1,2,4$ respectively. And Figures 4(d)-(e) demonstrate the results on CiaoDVD dataset. We plot the total opinion achieved by each compared method with a step size of 1 . For the best visualization of the results, we start the plot from the different number of the round for different choice of $k$. We can observe that the proposed CONE outperforms all compared methods in almost every round of MLT on both datasets. Even the degenerated version of our method Cone- can outperform the other three LT methods, which supports 


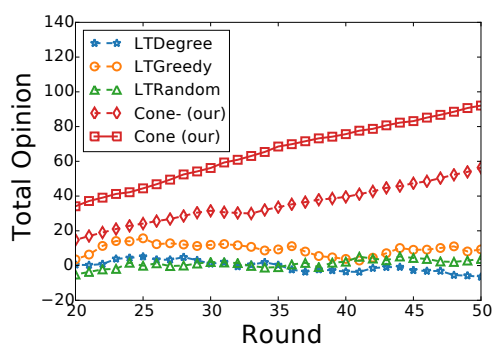

(a) Flixster $(k=1)$

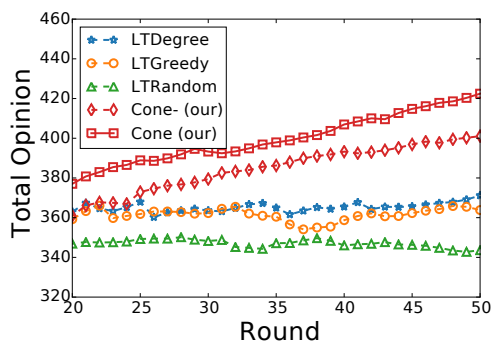

(d) $\operatorname{CiaoDVD}(k=1)$

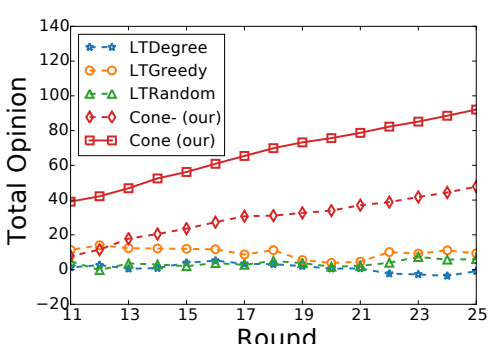

(b) Flixster $(k=2)$

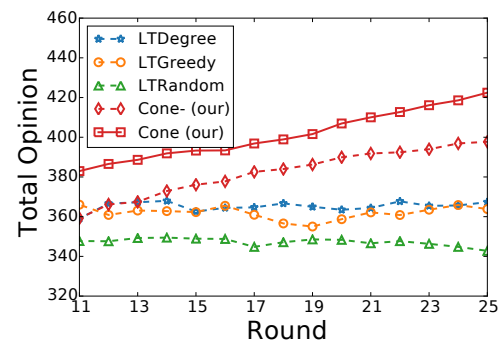

(e) CiaoDVD $(k=2)$

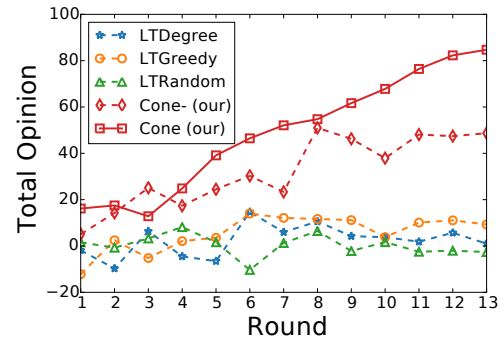

(c) Flixster $(k=4)$

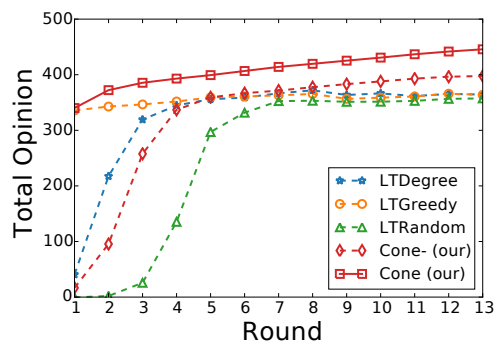

(f) $\operatorname{CiaoDVD}(k=4)$

Figure 4: Comparison of all methods in terms of total opinion.

the intuition that maximizing the number of influenced users may not achieve good total opinion. We further observe that ConE consistently outperforms CONE- in two datasets. These results suggest that considering opinion estimation and seeds selection collectively can improve the performance of opinion maximization. Interestingly, from Fig. 4(f) we can observe that the total opinion achieved by the compared methods are dramatically different even in the early stage of promotion. This is because the network of CiaoDVD is more densely connected than Flixster. And when we select a relatively large number of seeds for each round $(k=4)$, the influence propagates much faster.

In Table 2, we also present the results of the number of positive opinions versus the number of negative opinions achieved in the end of round $T$ for all compared methods All results in Table 2 is obtained with $k=1$, results with other settings of $k$ are similar and are omitted due to the page limitation. From Table 2 we can observe that although CONE cannot achieve the largest number of positive opinions, it can always achieve a lower spread of negative opinion than LTGREEDY and LTDEGREE. As we argued in Section 1, negative opinions hurt the reputation of the product and can make potential customers turn to the competitors and never come back. Thus, Cone is a better diffusion method for the company who want to optimize the profit by preventing negative opinions spread in the network. Furthermore, the column of \# pos. - \# neg. indicates the difference between the number of positive opinions and the number of negative achieved by each method. We can observe that CONE always outperform other methods on this metric, indicating CONE can better balance the positive opinions and negative opinions achieved in the network than other methods including CONE-, which again proves the advantage of considering opinion estimation and seeds selection collectively.

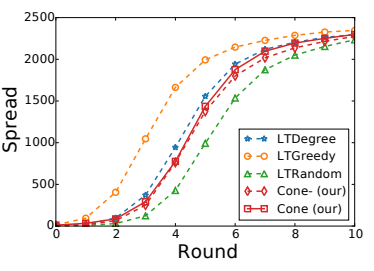

(a) Flixster

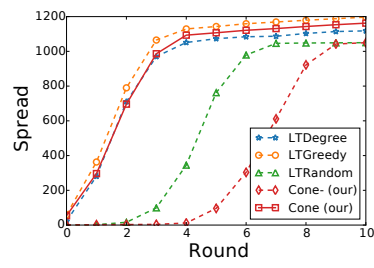

(b) CiaoDVD
Figure 5: Comparison of spread

\subsection{Comparison of Influence Spread}

We also present the comparison of influence spread for the compared methods in Fig. 5. In both datasets, ConE outperforms LTRANDOM and CONE- in terms of total spread, and achieves comparable or larger amount of spread to LTDEGREE with much better total opinion (as shown in Fig. 4). We further observe that CONE achieves a slightly slower spread of activated nodes than LTGREEDY. However, CONE can eventually reach the same level of total spread as LTGREEDY in the later stage of propagation. Larger spread indicates more adoption of the target product, which brings more profits to the company. Thus, we can conclude that CONE can achieve better total opinion with little compromise on the adoption.

Table 3: Comparison of Related Works.

\begin{tabular}{c|cccc}
\hline & data-driven & item-aware & multi-round & opinion \\
\hline$[6]$ & $\checkmark$ & & & \\
{$[2,5,23]$} & & & & $\checkmark$ \\
{$[13]$} & $\checkmark$ & & $\checkmark$ & \\
{$[1]$} & $\checkmark$ & $\checkmark$ & & \\
this paper & $\checkmark$ & $\checkmark$ & $\checkmark$ & $\checkmark$ \\
\hline
\end{tabular}




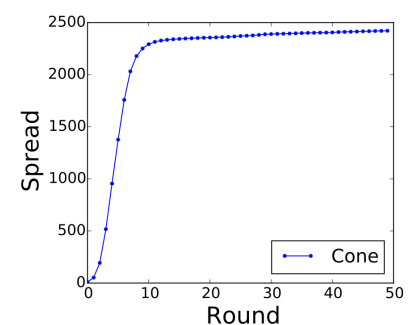

(a) Popular item $\left(r_{\text {avg }}=2.91\right)$

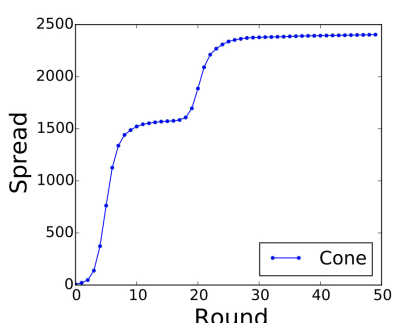

(b) Unpopular item $\left(r_{\text {avg }}=2.18\right)$

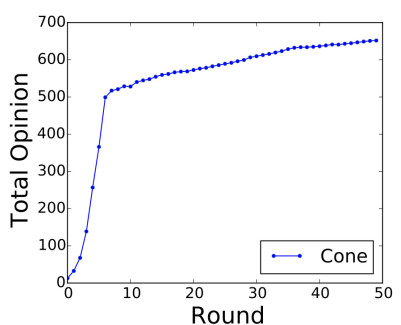

(c) Popular item $\left(r_{\text {avg }}=2.91\right)$

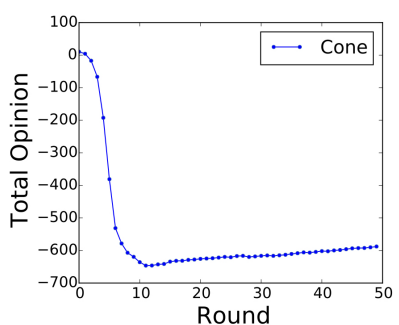

(d) Unpopular item $\left(r_{\text {avg }}=2.18\right)$

Figure 6: Case study on Cone.

\subsection{Case Study}

We select two items with different average ratings in Flixster dataset to present their spread and opinion achieved by using ConE method. Figure 6(a) shows the spread curve achieved by CoNE on a popular item (average rating $=2.91$ ) and the corresponding total opinion curve in shown in Figure 6(c). In contrast, Figure 6(b) shows the spread curve of CONE on an unpopular item (average rating $=2.18$ ) and and the corresponding total opinion curve in shown in Figure 6(d). We can observe that CONE is much more conservative when promoting an unpopular item than the case of promoting a popular item. The results indicate that CONE can adjust the promotion strategies according to the opinions observed in the network to optimize the selection of seed users.

\section{RELATED WORK}

Our work is related to influence maximization (viral marketing), opinion maxization and collaborative filtering, we briefly discuss them respectively in this section.

\subsection{Influence Maximization}

Influence maximization (i.e., viral marketing) was first studied by Domingos et al. [4]. Kempe et al. later studied this problem through social network[10], two propagation models, i.e., LT (Linear Threshold) model, and IC (Independent Cascade) model, are proposed to simulate the real-world scenario of influence diffusion. Later, Chen et al. proposed a scalable algorithm for IC model which can be easily applied on networks with millions of nodes [3]. Goyal et al. proposed a data-based approach [6] to model the influence maximization problem, instead of randomly generating the model parameters, their model learns influence-ability of users from the historical traces of action propagations. Barbieri et al. [1] studied the problem of topic-aware influence maximization based on the IC model, which distinguishes the ability of influence of users on different topics. Lin et al. considered the problem with multi-round and multi-party setting, in which two competitive players select their seeds to activate in turn to maximize their own outcomes [13]. Hung et al. proposed to study the problem of influence maximization with item inference, where they considered the association rule in influence propagation [8].

\subsection{Opinion Maximization}

Chen et al. [2] first proposed to incorporate opinion into influence maximization problem based on the IC model, and their objective is to maximize the total number of activated users with positive opinions. Zhang et al. [23] later proposed another adapted IC model to consider negative or neutral opinions. However, their model uses randomly generated opinions over the network and assumes the opinions are already known before the propagation starts. Gionis et al. [5] model the opinion maximization problem as a game between users in the social network, where each user holds a subjective opinion about a target product or idea, but the expressed opinions of each user is a trade-off between their own internal opinions and their neighbors' expressed opinions. All these works are neither data-driven nor item-aware, overlooking the abundant historical rating data.

\subsection{Collaborative Filtering}

The goal of collaborative filtering (CF) [11, 12, 14-16, 22, 25] is to make accurate recommendations to users based on their past reviews and feedback on existing products. Sarwar et al. proposed an item-based approach for CF problem, in which they utilize the similarity among items to make recommendation [16]. Koren et al. proposed to use low-rank matrix factorization method to solve the problem [12]. Yang et al. studied the CF problem with social links and trust [22]. The major difference between $\mathrm{CF}$ problem and the proposed Атом problem is that $\mathrm{CF}$ assumes all users are isolated and no influence is propagated from users to users.

\section{CONCLUSION}

We studied the Атом problem and proposed a novel method ConE to solve it. CONE is based on the MLt diffusion model, where new seed users are activated at the beginning of each round. ConE estimates the user opinion towards the target product and optimizes the seeds selection collectively at each round of MLт. The experimental results show that CONE improves the overall opinion spread in the network. An interesting future work for this topic is to study the Атом problem under the multi-party (or competitive) settings, in which multiple promoters are practicing the promotion simultaneously.

\section{ACKNOWLEDGEMENTS}

This work is supported in part by National Science Foundation through grant IIS-1718310, MRI-1626236, IIS-1526499, IIS-1763325, and CNS-1626432. This work is also supported in part by NSFC through grant 61672313. 


\section{REFERENCES}

[1] N. Barbieri, F. Bonchi, and G. Manco. 2012. Topic-Aware Social Influence Propagation Models. In ICDM '12. 81-90.

[2] W. Chen, A. Collins, R. Cummings, T. Ke, Z. Liu, D. Rincon, X. Sun, Y. Wang, W Wei, and Y. Yuan. 2011. Influence maximization in social networks when negative opinions may emerge and propagate. In SDM '11.379-390.

[3] W. Chen, C. Wang, and Y. Wang. 2010. Scalable influence maximization for prevalent viral marketing in large-scale social networks. In KDD '10. 1029-1038.

[4] P. Domingos and M. Richardson. 2001. Mining the network value of customers In $K D D$ '01. 57-66.

[5] A. Gionis, E. Terzi, and P. Tsaparas. 2013. Opinion maximization in social networks. In SDM '13. 387-395.

[6] A. Goyal, F. Bonchi, and L. Lakshmanan. 2011. A data-based approach to social influence maximization. In VLDB'11, Vol. 5. 73-84.

[7] G. Guo, J. Zhang, D. Thalmann, and N. Yorke-Smith. 2014. ETAF: An Extended Trust Antecedents Framework for Trust Prediction. In ASONAM '14.

[8] H. Hung, H. Shuai, D. Yang, L. Huang, W. Lee, J.Pei, and M. Chen. 2016. When Social Influence Meets Item Inference. In KDD '16. 915-924.

[9] M. Jamali and M. Ester. 2010. A matrix factorization technique with trust propagation for recommendation in social networks. In RecSys '10. 135-142.

[10] D. Kempe, J. Kleinberg, and É. Tardos. 2003. Maximizing the spread of influence through a social network. In KDD '03. 137-146.

[11] Y. Koren. 2009. Collaborative filtering with temporal dynamics. In $K D D$ '09. 447-456.

[12] Y. Koren, R. Bell, and C. Volinsky. 2009. Matrix factorization techniques for recommender systems. Computer 42, 8 (2009).
[13] S. Lin, S-D. Lin, and M. Chen. 2015. A learning-based framework to handle multi-round multi-party influence maximization on social networks. In KDD '15. 695-704.

[14] X. Liu, C. Aggarwal, Y. Li, X. Kong, X. Sun, and S. Sathe. 2016. Kernelized matrix factorization for collaborative filtering. In $S D M$ '16. 378-386.

[15] X. Liu, Y. Song, C. Aggarwal, Y. Zhang, and X. Kong. 2017. BiCycle: Item Recommedation with Life Cycles. In ICDM '17. 297-306.

[16] B. Sarwar, G. Karypis, J. Konstan, and J. Riedl. 2001. Item-based collaborative filtering recommendation algorithms. In $W W W^{\prime} 01.285-295$.

[17] M. Solomon. 2014. Consumer behavior: Buying, having, and being. Vol. 10. Prentice Hall Engelwood Cliffs, NJ

[18] X. Song, Y. Chi, K. Hino, and B. Tseng. 2007. Information flow modeling based on diffusion rate for prediction and ranking. In $W W W^{\prime}$ '07. 191-200.

[19] J. Sun and J. Tang. 2011. A survey of models and algorithms for social influence analysis. In Social Network Data Analytics. Springer, 177-214.

[20] J. Tang, J. Sun, C. Wang, and Z. Yang. 2009. Social influence analysis in large-scale networks. In $K D D$ '09. 807-816.

[21] S. Wasserman and K. Faust. 1994. Social network analysis: Methods and applications. Vol. 8. Cambridge University Press.

[22] B. Yang, Y. Lei, D. Liu, and J. Liu. 2013. Social collaborative filtering by trust. In IfCAI'13. 2747-2753.

[23] H. Zhang, T. Dinh, and M. Thai. 2013. Maximizing the spread of positive influence in online social networks. In ICDCS'13. 317-326.

[24] J. Zhang, S. Wang, Q. Zhan, and P. Yu. 2016. Intertwined Viral Marketing in Social Networks. In ASONAM '16.

[25] K. Zhou, S. Yang, and H. Zha. 2011. Functional matrix factorizations for cold-start recommendation. In SIGIR '11. 315-324. 